\title{
AMPEROMETRIC DETERMINATION OF ENDOGLIN IN HUMAN SERUM USING DISPOSABLE IMMUNOSENSORS CONSTRUCTED WITH POLY(PYRROLEPROPIONIC) ACID-MODIFIED ELECTRODES
}

E. Martínez-Periñán ${ }^{\mathrm{a}}$, E. Sánchez-Tirado ${ }^{\mathrm{a}}$, A. González-Cortés ${ }^{\mathrm{a}}$, R. Barderas ${ }^{\mathrm{b}}$, J.M. SánchezPuelles $^{c}$, L. Martínez-Santamaría ${ }^{\text {, }}$, S. Campuzano ${ }^{a}$, P. Yáñez-Sedeño a,*, J.M. Pingarrón ${ }^{\text {a }}$.

${ }^{a}$ Department of Analytical Chemistry, Faculty of Chemistry, University Complutense of Madrid, 28040-Madrid, Spain

${ }^{\mathrm{b}}$ UFIEC, CROSADIS, National Institute of Health Carlos III, Majadahonda, 28222-Madrid, Madrid, Spain

${ }^{\mathrm{c}}$ Departamento de Medicina Celular y Molecular, Centro de Investigaciones Biológicas, Consejo Superior de Investigaciones Científicas, 28040-Madrid, Spain

${ }^{\mathrm{d}}$ Department of Bioengineering, UC3M; CIEMAT; IIS-Fundación Jiménez Díaz; CIBER de Enfermedades Raras (CIBERER), Madrid, Spain.

\footnotetext{
* To whom correspondence should be addressed
} 


\section{ABSTRACT}

An amperometric immunosensor for the determination of the biomarker endoglin (CD105) to comply with the requirements of sensitivity and accuracy demanded in clinical practice is reported in this work. The immunosensing platform is implemented onto disposable electrodes modified with poly(pyrrolepropionic) acid (pPPA). The methodology involves a sandwich configuration and labeling of the biotinylated detector antibody with poly-HRPstreptavidin for signal amplification. Amperometric detection of hydrogen peroxide reduction in the presence of HQ was employed as analytical readout. The different steps involved in the immunosensor preparation were monitored by electrochemical impedance spectroscopy. The resulting immunosensor provided a linear range between 0.18 and $20 \mathrm{ng} \mathrm{mL}^{-1}$, adequate for the determination of CD105 in serum, with a detection limit (LOD) of $140 \mathrm{pg} \mathrm{mL}^{-1}$. These analytical characteristics improve those reported previously for other electrochemical immunosensors. A comparison with immunosensors prepared using other electrochemical scaffolds for anti-CD105 immobilization involving carboxylated doubled walled carbon nanotubes or modification by grafting, showed that a better performance was achieved using pPPA-modified SPCEs. A good reproducibility of the measurements, an excellent storage stability of the anti-CD105-pPPA/SPCE bioplatforms and an excellent selectivity of the resulting immunosensors were found. The usefulness of the immunosensors was tested by analyzing human serum samples collected from healthy individuals and patients of colorectal, breast and lung cancer and epidermolysis bullosa. The results were successfully validated against those provided by an ELISA kit.

Keywords: Endoglin, CD105, human serum, electrochemical immunosensor, serum. 


\section{Introduction}

Human endoglin (CD105) is a $180 \mathrm{kDa}$ homodimeric hypoxia-inducible cell transmembrane type III glycoprotein, densely expressed on the surface of angiogenic proliferating endothelial cells that acts as an auxiliary receptor for the transforming growth factor (TGF) family of cytokines. This protein plays a significant role in angiogenesis, a crucial process involved in several physiological and pathological conditions such as cancer $[1,2]$. The stronger CD105 expression found in a wide range of endothelial tumors, including colon, breast, brain, lung, prostate and cervical cancer, compared to normal tissues, suggests the possible involvement of CD105 in tumor angiogenesis [3-5]. In fact, increasing levels of CD105 in biological fluids from affected patients may be used as an indicator for disease progression and risk of metastasis [6,7]. CD105 circulating levels have been found to be altered in response to chemotherapy and, therefore, their monitoring can be useful for both the evaluation of the response of patients to treatment, especially to antiangiogenic therapies, and in tracing disease recurrence.

Increased CD105 levels are also detected in inflamed tissues. The protein modulates TGF- $\beta$ induced production [8] and, therefore, is not involved only in cancer but also in the development and progression of other important diseases related to angiogenesis such as preeclampsia [9], rheumatoid arthritis [10] and skin diseases such as epidermolysis bullosa $[11]$.

Various immunoassay methods for the detection of CD105 have been reported so far [12] related with cancer treatment [2] or diagnostics of preeclampsia [13]. ELISA kits available from Thermo Scientific [14] or Sigma Aldrich [15] are examples of commercial kits involving sandwich-type configurations with anti-CD105 capture antibodies, biotinylated anti-CD105, and peroxidase (HRP)-streptavidin conjugates. These kits provide dynamic ranges extending from units to thousands of $\mathrm{pg} \mathrm{mL}^{-1}$ with minimum detectable doses (MDD), 
calculated statistically from the zero signal value, of units of $\mathrm{pg} \mathrm{mL}^{-1}$. An important disadvantage of these methods is the long analysis time required (five hours). More simple immunoassays involving capture and HRP-labeled detection antibody as immunoreagents are also available. For instance, the ELISA kit from Sino Biologicals [16] provides a calibration plot ranging from 62.5 to $4,000 \mathrm{pg} \mathrm{mL}^{-1} \mathrm{CD} 105$ with a MDD value of $36 \mathrm{pg} \mathrm{mL}^{-1}$ in an assay time of $3 \mathrm{~h} 40 \mathrm{~min}$.

Regarding electrochemical immunosensors, to our knowledge only two configurations have been reported so far. A sandwich type immunosensor involved immobilization of antiCD105 capture antibody on a mercaptoethylamine self-assembled monolayer (SAM) onto a gold nanoparticles (AuNPs)-modified gold electrode and the use of a detection antibody chemically linked to the electron mediator thionine and platinum nanoparticles (PtNPs) for signal amplification [17]. This approach provided a linear range from 1.3 to $200.0 \mathrm{ng} \mathrm{mL}^{-1}$ and a detection limit (LOD) of $0.9 \mathrm{ng} \mathrm{mL}$. However, it implies laborious and time consuming protocols for SAM modification of the electrode surface $(26 \mathrm{~h})$ and preparation of the labeled immunoconjugates (12.5 h). More recently, a sandwich-type immunosensor was implemented on the surface of magnetic microbeads (MBs) and used capture and HRPdetection antibodies as well as amperometric detection of hydrogen peroxide in the presence of hydroquinone (HQ). A linear relationship between 0.8 and $10.0 \mathrm{ng} \mathrm{mL}^{-1}$ and a LOD of 0.2 $\mathrm{ng} \mathrm{mL} \mathrm{m}^{-1}$ were achieved [18]. It is worth to mention that although both immunosensors provide higher LODs than ELISA kits, the sensitivity is sufficient for the determination of the target protein in serum of patients diagnosed with cancer, where the mean values found using a radioimmunoassay were of $34.0 \pm 26.8$ and $63.8 \pm 72.5 \mathrm{ng} \mathrm{mL}^{-1}$ in metastasis-negative (42) and positive (59) patients, respectively [19]. However, the great variability observed in these results highlights the need for developing methodologies that provide not only good sensitivity but also good reproducibility for the determination of this circulating biomarker. 
Nowadays the use of electrodes modified with electropolymerized conducting polymers containing suitable functional groups offers an interesting strategy to develop electrochemical immunosensing platforms with enhanced performance. For example, poly(pyrrolepropionic) acid (pPPA), a conducting polymer with abundance of carboxyl groups, allows the immobilization of large bioreagents loadings and permeation of electroactive species to the electrode surface due to its porous structure [20]. Despite these interesting properties, only few examples of electrochemical immunosensors involving the use of pPPA as modifier of glassy carbon electrodes [21-24] and SPCEs [25] have been found in the literature.

In this work, a strategy able to comply with the requirements of sensitivity and accuracy demanded by the determination of CD105 is described. The immunosensing platform was implemented onto a disposable electrode modified with pPPA and involved a sandwich configuration as well as labeling of the biotinylated detector antibody with poly-HRPstreptavidin for signal amplification. Amperometric detection of hydrogen peroxide reduction in the presence of HQ was employed as analytical readout. The immunosensor provided a calibration plot with a linear range between 0.18 and $20 \mathrm{ng} \mathrm{mL}^{-1} \mathrm{CD} 105$, a LOD value of 140

$\mathrm{pg} \mathrm{mL} \mathrm{m}^{-1} \mathrm{CD} 105$ and was successfully applied to the determination of the target analyte in serum samples from cancer or epidermolysis bullosa patients with minimal sample treatment.

\section{Experimental}

\subsection{Apparatus and electrodes}

Screen-printed carbon electrodes (SPCEs, 110 DRP,) were used as electrochemical transducers (DropSens-Metrohm (Oviedo, Spain). They are constituted of a $4 \mathrm{~mm} \emptyset$-working electrode, a silver pseudo-reference electrode and a carbon counter electrode. A $\mu$-Autolab type III potentiostat, operated by the GPES 4.7 software (EcoChemie), was employed for electropolymerization of PPA onto SPCEs. Electrochemical impedance spectroscopy (EIS) 
was carried out using an Autolab type III potentiostat controlled by the FRA2 software (EcoChemie). Amperometric measurements were performed with an INBEA Biosensores S.L. potentiostat using the Ib-Graph software. An Elmasonic Se60 (Elma) ultrasonic bath, a Vortex homogenizator (Velp Scientifica) and a Crison Basic 20+ pH-meter were also used. All electrochemical experiments were performed at room temperature.

\subsection{Reagents and solutions}

Mouse anti-human endoglin capture antibody (anti-CD105), from Human Endoglin/CD105 DuoSet Elisa Catalog Number: DY1097 from R\&D Systems, was reconstituted with $1.0 \mathrm{~mL}$ 0.01 M phosphate buffer saline solution (PBS) of pH 7.4 containing $137 \mathrm{mM} \mathrm{NaCl}, 2.7 \mathrm{mM} \mathrm{KCl}, 8.1 \mathrm{mM} \mathrm{Na}_{2} \mathrm{HPO}_{4}$ and $1.5 \mathrm{mM} \mathrm{KH}_{2} \mathrm{PO}_{4}$. Anti-CD105 solutions were prepared by appropriate dilution with $25 \mathrm{mM}$ 2-( $N$-morpholino)ethanesulfonic acid (MES, from Gerbu) buffer solution of pH 5.0. CD105 standard solutions and biotinylated goat anti-human endoglin (Biotin-anti-CD105), also from Human Endoglin/CD105 DuoSet Elisa, Catalog Number: DY1097 from R\&D Systems, were prepared in 0.01 M PBS solution of pH 7.4 supplemented with $1 \%(\mathrm{w} / \mathrm{v})$ BSA.

Pyrrole propionic acid (PPA) (Sigma-Aldrich, 97\%) and KCl (Scharlau, 99.5\%) were used. The electro-synthesis of pPPA polymer was made from $5 \mathrm{mM} \mathrm{PPA}+0.5 \mathrm{M} \mathrm{KCl}$ aqueous solutions. N-(3-dimethyl-aminopropyl)-N'-ethylcarbodiimide (EDC, Acros Organics) and N-hydroxysulfo-succinimide (Sulfo-NHS, Sigma-Aldrich) were used as activation agents for carboxyl groups confined at the electrode surface. $50 \mathrm{mg} \mathrm{mL}^{-1}$ solutions of EDC and Sulfo-NHS each in $25 \mathrm{mM}$ MES buffer of $\mathrm{pH} 5$ were used for this purpose. The blocking agent was a commercial $1 \%(\mathrm{w} / \mathrm{v})$ casein solution prepared in $0.01 \mathrm{M}$ PBS of $\mathrm{pH} 7$ (Blocker ${ }^{\mathrm{TM}}$ Casein, Thermo Fisher). Solutions of streptavidin labeled with horseradish

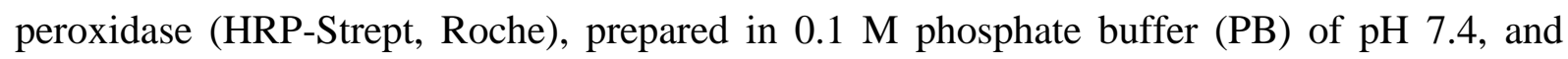
poly-HRP-Strept (85-R200), diluted in stabilizer solution (Fitzgerald), were also used. 
Hydrogen peroxide (Aldrich, 30\% (w/w)) and hydroquinone (HQ, Sigma) were also employed. $0.05 \mathrm{M} \mathrm{K}_{3} \mathrm{Fe}(\mathrm{CN})_{6}$ and $\mathrm{K}_{4} \mathrm{Fe}(\mathrm{CN})_{6}$ (Sigma) solutions were prepared in $0.01 \mathrm{M} \mathrm{PB}$ of $\mathrm{pH}$ 7.4. Bovine serum albumin (BSA), immunoglobulin $\mathrm{G}$ (IgG), hemoglobin (HB), uric acid (UA) and ascorbic acid (AA), all from Sigma, transforming growth factor beta-1 (TGF$\beta 1)$, interleukin 1 beta (IL-1 $\beta)$, E-cadherin (E-Cad), cadherin 17R (Cad17R), and fibroblast growth factor receptor 4 (FGFR4), all from R\&D System Inc., interleukin 6 (IL-6, Abcam), recombinant human ErbB2 protein (ERBB2, Sino Biological Inc.), and recombinant full length human p53 protein (p53, EMD Millipore Corporation), were tested as potential interfering compounds. Solutions of these reagents were prepared in 0.01 M PBS of pH 7.4. All other chemicals used were of analytical reagent grade. All solutions were prepared with deionized water obtained from a Milli-pore Milli-Q purification system $(18.2 \mathrm{M} \Omega \mathrm{cm}$ at 25 $\left.{ }^{\circ} \mathrm{C}\right)$.

\subsection{Procedures}

\subsubsection{Preparation of anti-CD105-pPPA/SPCE immunosensor}

Electropolymerization of pPPA on the SPCE was carried out by immersing the electrode in the $5 \mathrm{mM} \mathrm{PPA}+0.5 \mathrm{M} \mathrm{KCl}$ solution and applying 20 successive voltammetric cycles between 0 and $+0.85 \mathrm{~V}$ vs Ag pseudo reference electrode at $100 \mathrm{mV} \mathrm{s}^{-1}$. The resulting pPPA/SPCE was immersed into a $1 \mathrm{mM}$ HQ solution in $0.1 \mathrm{M} \mathrm{PB}$ of $\mathrm{pH} 7.4$, and a control cyclic voltammogram between -0.2 and $+1.0 \mathrm{~V}$ vs. the Ag pseudo-reference electrode at 50 $\mathrm{mV} \mathrm{s}^{-1}$ was recorded. Thereafter, electrode surface confined carboxyl groups were activated by adding $10 \mu \mathrm{L}$ of EDC/NHSS solution and allowing incubation for $30 \mathrm{~min}$ at $25^{\circ} \mathrm{C}$ in a humidified chamber. The electrode was washed with $25 \mathrm{mM}$ MES buffer of $\mathrm{pH} 5$, and the anti-CD105 capture antibody was covalently attached to the activated pPPA/SPCE by dropping $5 \mu \mathrm{L}$ of a $12.5 \mu \mathrm{g} \mathrm{mL}^{-1}$ antibody solution allowing incubation during $60 \mathrm{~min}$ at $25^{\circ} \mathrm{C}$. After washing with MES buffer, the unreacted activated groups on the anti-CD105- 
pPPA/SPCEs were blocked with $10 \mu \mathrm{L}$ of $1 \%$ casein solution during $30 \mathrm{~min}$. The antiCD105-pPPA/SPCEs were washed with $0.1 \mathrm{M} \mathrm{PB}$ of $\mathrm{pH} 7.4$, dried and used or stored at $20^{\circ} \mathrm{C}$ in dry conditions.

\subsubsection{Determination of CD105}

$5 \mu \mathrm{L}$ of the antigen solution (or the sample) were added onto the anti-CD105pPPA/SPCEs and incubated for $30 \mathrm{~min}$. Thereafter, $5 \mu \mathrm{L}$ of a $2 \mu \mathrm{g} \mathrm{mL} \mathrm{L}^{-1}$ Biotin-anti-CD105 solution were dropped onto the bioelectrode, incubated during $60 \mathrm{~min}$, and washed with 0.1 M PB of pH 7.4. Subsequently, $5 \mu \mathrm{L}$ of a 1:500 diluted poly-HRP-Strept solution were added and incubated for $15 \mathrm{~min}$. After washing with $0.1 \mathrm{M} \mathrm{PB}$ of $\mathrm{pH} 7.4$, a drop of this buffer was added on the resulting immunosensor and stored until use at room temperature. The determination of CD105 was accomplished by adding $45 \mu \mathrm{L}$ of a $1 \mathrm{mM}$ HQ solution (freshly prepared in $50 \mathrm{mM} \mathrm{PB}$ of $\mathrm{pH} 6.0$ ) on the surface of the horizontally positioned poly-HRPStrept-Biotin-anti-CD105-CD105-anti-CD105-pPPA/SPCE immunosensor and applying a potential of -200 mV vs the Ag pseudo-reference electrode. Once the background current was stabilized (100 s approximately), $5 \mu \mathrm{L}$ of a $50 \mathrm{mM} \mathrm{H}_{2} \mathrm{O}_{2}$ solution freshly prepared in the same buffer were added and incubated for $200 \mathrm{~s}$. Then, the cathodic current arising from the electrochemical reduction of benzoquinone was measured.

\subsubsection{Analysis of serum samples}

Serum samples were collected from patients affected by different types of cancer (colorectal, breast and lung cancer), as well as by epidermolysis bullosa. Epidermolysis bullosa patients were selected for age (18-40 years) and for being carriers of identical C7 mutation c.6527insC in exon 80 of the COL7A1 gene. The samples as well as control sera from healthy individuals were provided by Hospital Universitario La Paz and Clínico San Carlos (Madrid, Spain) with informed consent approved by the patients and Ethics Committee 
of the Institutions (in case of epidermolysis bullosa samples, Code HULP: PI-1602, which adhered to Helsinki Guidelines and further reviews, Fortaleza 2013, http://www.wma.net).

The samples were analyzed using poly-HRP-Strept-Biotin-anti-CD105-CD105-antiCD105-pPPA/SPCE immunosensors after a fifty-fold sample dilution in 0.01 M PBS of pH 7.4. Validation of the method was performed by comparison of the obtained results with those provided by applying a conventional ELISA methodology using the same immunoreagents.

\section{Results and discussion}

Fig. 1 shows schematically the different steps implied in the preparation of the polyHRP-Strept-Biotin-anti-CD105-CD105-anti-CD105-pPPA/ SPCE immunosensors as well as the amperometric measurement principle. Upon electropolymerization of PPA on the SPCE by cyclic voltammetry, the anti-CD105 was covalently immobilized through carbodiimide/succinimide chemistry on the pPPA/SPCE (a) and the remaining free active sites were blocked with casein (b). A sandwich type immunoassay involving CD105 (c), Biotin-anti-CD105 (d) and poly-HRP-Strept (d) was then implemented. The affinity reaction was amperometrically monitored by adding $\mathrm{H}_{2} \mathrm{O}_{2}$ in the presence of $\mathrm{HQ}$ as the redox mediator.

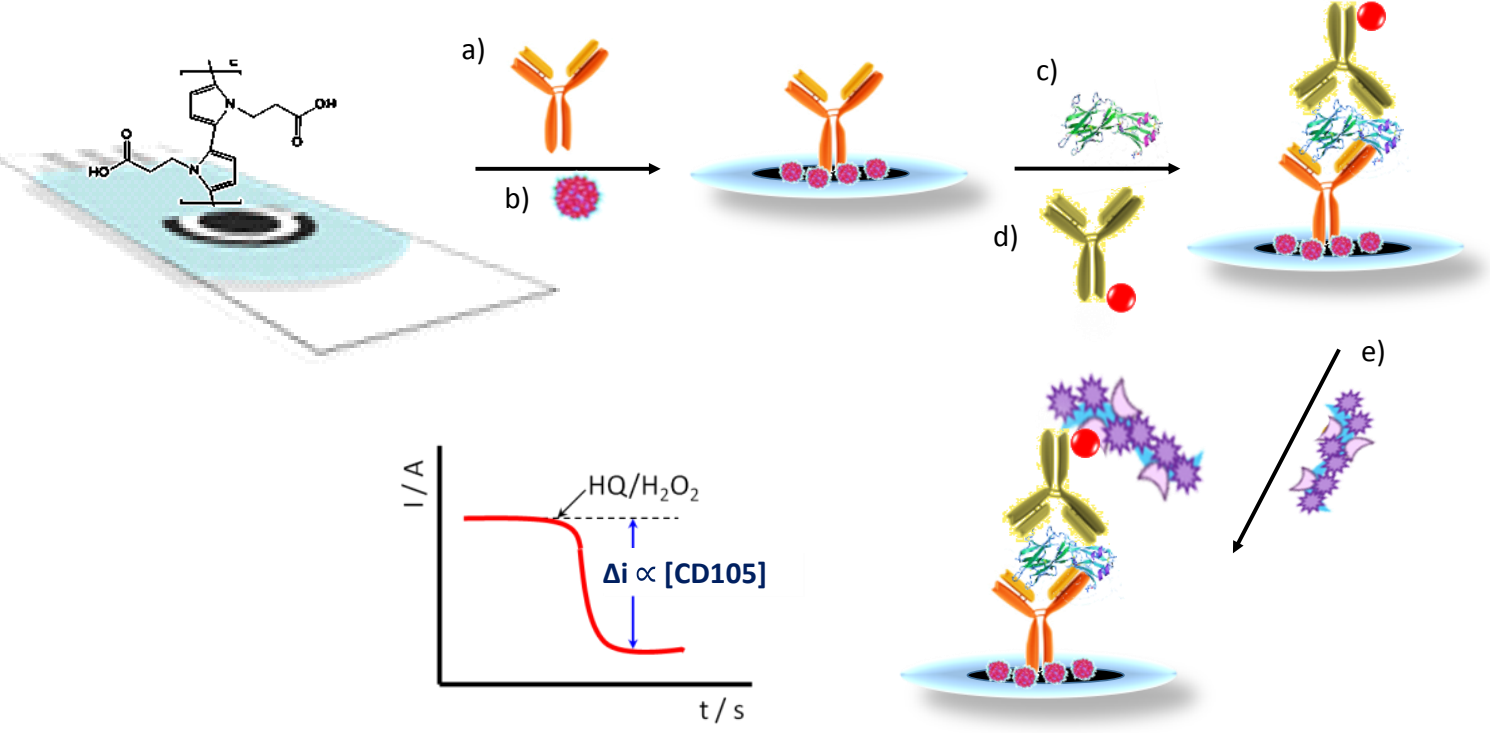


Fig. 1. Schematic display of the different steps involved in the preparation of the amperometric immunosensor for CD105 using pPPA-modified SPCEs: covalent immobilization of anti-CD105 (a), blocking step with casein (b), sandwich configuration using CD105 (c), Biotin-anti-CD105 (d), and poly-HRP-Strept conjugate (e).

\subsection{Optimization of the experimental variables involved in the preparation of the} immunosensor

The effect of different variables on the immunosensor response was evaluated. The tested variables were: anti-CD105 antibody loading on pPPA/SPCE and incubation time (a); Biotin-anti-CD105 loading on anti-CD105-pPPA/SPCE and incubation time (b); type and concentration of blocking agent (c); type of enzymatic tracer to label the Biotin-anti-CD105 conjugate (d) and poly-HRP-Strept concentration (e). The largest ratio between the currents measured in the presence $(\mathrm{S})$ or in absence $(\mathrm{N})$ of the target compound was taken as the selection criterion for each tested variable. Other variables involved in the immunosensor preparation such as the experimental conditions for pPPA electrodeposition onto SPCE were taken from previous work [25]. Furthermore, the conditions at which the amperometric currents from benzoquinone (BQ) generated by the HRP reaction using the $\mathrm{H}_{2} \mathrm{O}_{2} / \mathrm{HQ}$ system, were taken also from a previous work [26].

The results of the optimization studies are summarized in Table 1. Details are given in the Supporting Information and Figs. S1-S4.

\section{Table 1}

Optimization of the experimental variables affecting the performance of poly-HRP-Streptanti-CD105-CD105-anti-CD105-pPPA/SPCE immunosensor for the amperometric determination of CD105. 


\begin{tabular}{|c|c|c|}
\hline Variable & Range (or compound) tested & Selected \\
\hline Anti-CD105 loading, $\mu \mathrm{g} \mathrm{mL}^{-1}$ & $5-17.5$ & 12.5 \\
\hline Incubation time for anti-CD105, min & $30-75$ & 60 \\
\hline Biotin-anti-CD105 loading, $\mu \mathrm{g} \mathrm{mL} \mathrm{L}^{-1}$ & $1-4$ & 2 \\
\hline $\begin{array}{l}\text { Incubation time for Biotin-anti- } \\
\qquad \text { CD105, min }\end{array}$ & $30-75$ & 45 \\
\hline Type of blocking agent & casein, BSA & casein \\
\hline \multicolumn{3}{|l|}{ Concentration of blocking agent, $\%$} \\
\hline$(w / v)$ & $1-2$ & 1 \\
\hline Incubation time for casein, min & $15-75$ & 30 \\
\hline Enzymatic tracer to label the Biotin- & HRP-Strept, & poly-HRP- \\
\hline anti-CD105 & poly-HRP-Strept & Strept \\
\hline Poly-HRP-Strept dilution & $1 / 1,000-1 / 250$ & $1 / 500$ \\
\hline
\end{tabular}

Once the experimental variables were optimized, the different steps involved in the immunosensor preparation were monitored by electrochemical impedance spectroscopy (EIS) using $2 \mathrm{mM} \mathrm{Fe}(\mathrm{CN})_{6}{ }^{4-/ 3-}$ as the redox probe in $0.05 \mathrm{M}$ PB of $\mathrm{pH} 6.0$ (Fig. 2). As expected, the modification of the SPCE with pPPA gave rise to a large increase in the electron transfer resistance, $\mathrm{R}_{\mathrm{CT}}$, varying from $2,383 \Omega$ (bare $\mathrm{SPCE}$ ) to $5,887 \Omega$ (pPPA/SPCE) as a consequence of two effects: a) the relatively low conductivity of the polymer and b) the electrostatic repulsion between the redox probe and the negatively charged carboxylate groups at the working $\mathrm{pH}$. Activation with EDC/NHSS provoked a dramatic decrease of the $\mathrm{R}_{\mathrm{CT}}$ value $(1,331 \Omega)$ which can be explained by the neutralization of the negative charge of the surface confined $-\mathrm{COOH}$ groups after the activation reaction. A further increase in the $\mathrm{R}_{\mathrm{CT}}$ value was observed after conjugation with anti-CD105 $(2,180 \Omega)$ due to the partially 
insulating barrier on the electrode surface produced by the presence of the antibody. As expected, blocking with casein of the remaining unreacted sites led to an increase in the $R_{C T}$ value $(2,246 \Omega)$, and the subsequent incorporation of the antigen provoked a slightly higher $\mathrm{R}_{\mathrm{CT}}$ value $(2,354 \Omega)$.

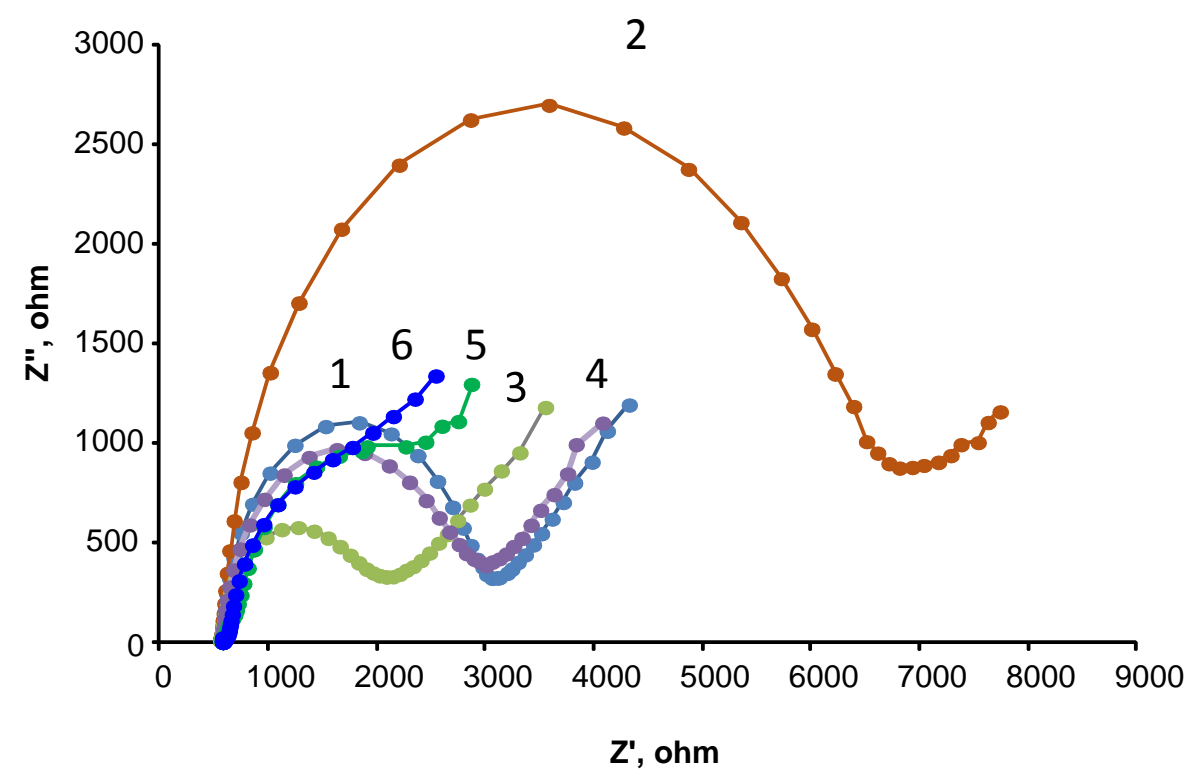

Fig. 2. Nyquist plots recorded in $2 \mathrm{mM}\left[\mathrm{Fe}(\mathrm{CN})_{6}\right]^{3-/ 4-}$ in $0.05 \mathrm{M}$ phosphate buffer solution of pH 6.0 at: SPCE (1); pPPA/SPCE (2); EDC/NHSS-activated pPPA/SPCE (3); anti-CD105pPPA/SPCE (4); casein/anti-CD105-pPPA/SPCE (5); CD105-anti-CD105-pPPA/SPCE (6); 0.04-100,000 Hz frequency range with a $10 \mathrm{mV}$ r.m.s. signal.

\subsection{Analytical figures of merit of the immunosensor}

Fig. 3 displays the calibration plot for CD105 standard solutions constructed with the developed immunosensor under the optimized working conditions. Error bars were calculated from measurements carried out with three different immunosensors. Some typical recorded amperograms are also displayed in Fig. 3. The steady state current vs. logarithm of CD105 concentration followed the adjusted equation $\Delta \mathrm{i}, \mathrm{nA}=(476 \pm 12) \log [\mathrm{CD} 105], \mathrm{ng} \mathrm{mL}^{-1}-$ $(406 \pm 1)\left(r^{2}=0.996\right)$, where $\Delta \mathrm{i}$ is the difference between the currents measured in the presence and in the absence of capture antibody [25]. The range of linearity extended between 0.18 and 
$20 \mathrm{ng} \mathrm{mL}^{-1}$ covering more than two orders of magnitude and is adequate for the determination of CD105 in serum according to the expected concentrations, at the $\mathrm{ng} \mathrm{mL}^{-1}$ level, in this type of sample [19], The LOD, $140 \mathrm{pg} \mathrm{mL}^{-1}$, was calculated according to the $3 \times \mathrm{s}_{\mathrm{b}}$ criterion, where $\mathrm{s}_{\mathrm{b}}$ was estimated as the standard deviation, expressed in concentration units, calculated from ten amperometric responses of the immunosensor in the absence of CD105. It is worth to mention that these analytical characteristics improve those reported by Zeng et al. with a LOD of $900 \mathrm{pg} \mathrm{mL}^{-1}$ [17], and are also slightly better than those obtained with an immunosensor using MBs [18] that exhibited a linear calibration plot from 0.8 to $10 \mathrm{ng} \mathrm{mL}^{-1}$ and a LOD of $200 \mathrm{pg} \mathrm{mL}^{-1} \mathrm{CD} 105$.
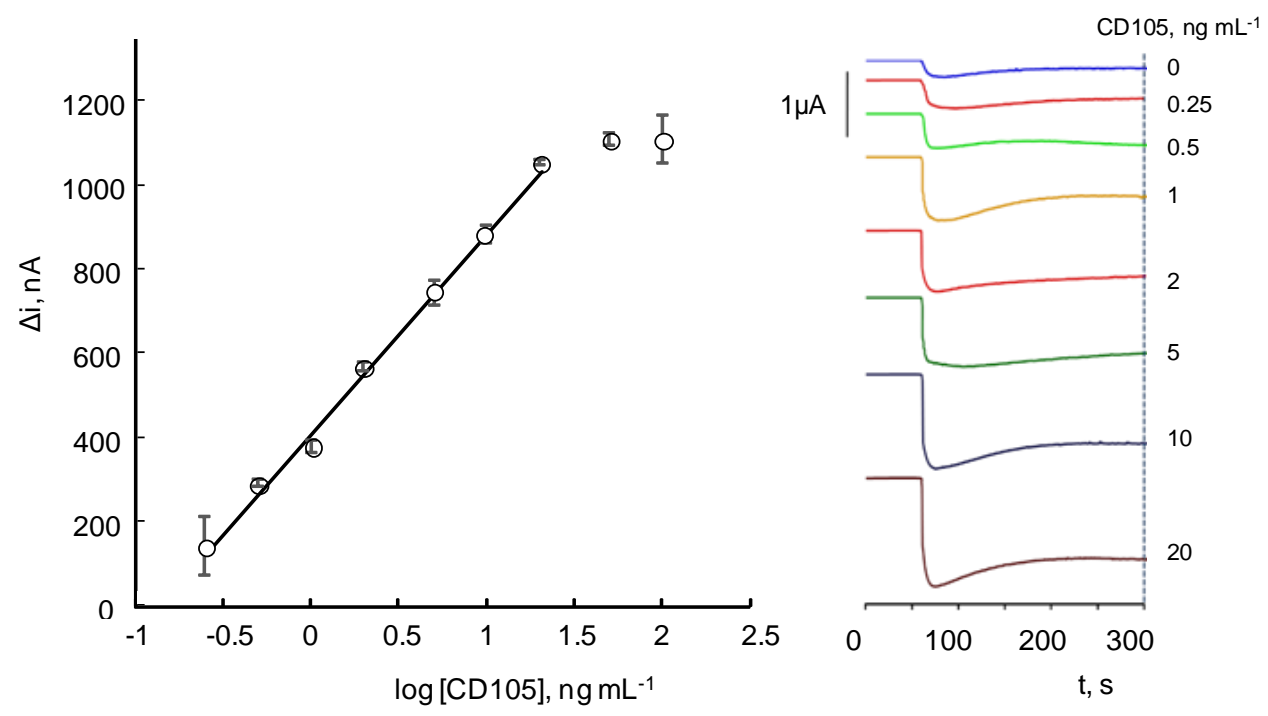

Fig. 3. Calibration plot (left) and amperograms recorded for the determination of CD105 standards at the poly-HRP-Strept-Biotin-anti-CD105-CD105-anti-CD105-pPPA/SPCE. Error bars estimated as triple of the standard deviation $(n=3)$.

The amperometric responses measured with five different immunosensors prepared on the same day for 0 and $1 \mathrm{ng} \mathrm{mL} \mathrm{m}^{-1} \mathrm{CD} 105$ yielded relative standard deviation (RSD) values of $3.8 \%$ and $5.2 \%(\mathrm{n}=5)$, respectively. Moreover, the same measurements with five different immunosensors constructed on different days gave RSD values of $4.9 \%$ and $5.7 \%(n=5)$, 
respectively, thus showing that the protocols used for the biosensor fabrication and the amperometric detection are suitable to allow a good reproducibility of the measurements.

The storage stability of anti-CD105-pPPA/SPCE bioelectrodes once the blocking step with casein was made, was also tested. Different anti-CD105-pPPA/SPCEs were prepared on the same day, stored under dry conditions at $-20^{\circ} \mathrm{C}$, and employed to prepare immunosensors to measure $20 \mathrm{ng} \mathrm{mL}^{-1} \mathrm{CD} 105$ each control day. The control chart shown in Fig. 4 indicates that the immunosensor responses remained within the control limits, set at $\pm 3 \mathrm{~s}$, were $\mathrm{s}$ was the standard deviation of the measurements carried out with 10 different immunosensors prepared the day 0 of this study, for at least 43 days (no longer storage times were tested). These results demonstrated the excellent stability of the anti-CD105-pPPA/SPCE bioplatforms.

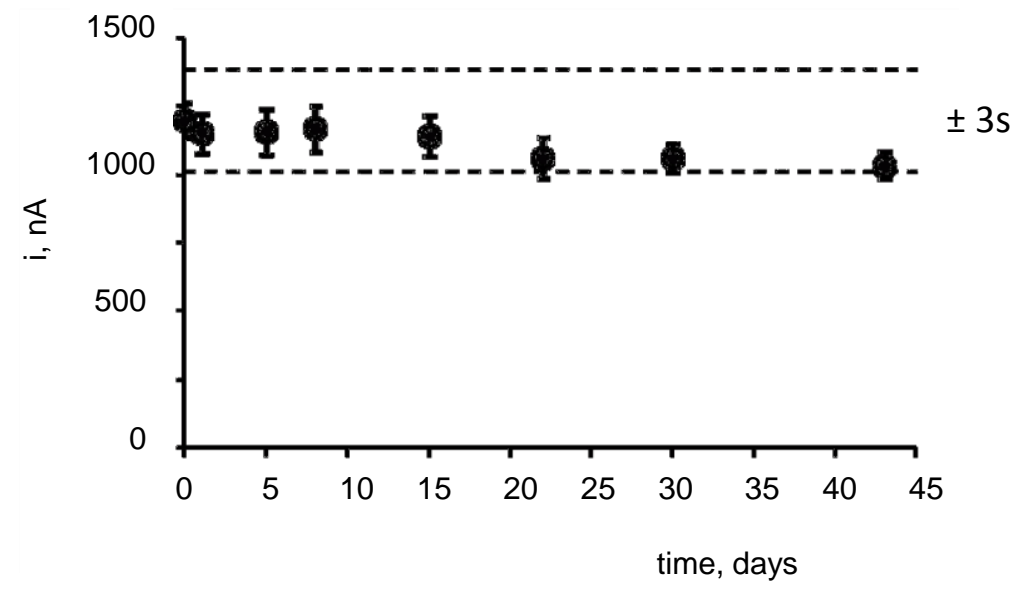

Fig. 4. Control chart constructed to check the storage stability of anti-CD105-pPPA/SPCE bioelectrodes upon storage at $-20^{\circ} \mathrm{C}$ under dry conditions. The central value was the average amperometric signals provided by 10 different immunosensors prepared the day 0 of the study for $20 \mathrm{ng} \mathrm{mL}^{-1} \mathrm{CD} 105$. Upper and lower limits of control were set as three times the standard deviation $(3 \mathrm{~s})$ of these measurements. Error bars estimated as triple of the standard deviation $(n=3)$. 


\subsection{Selectivity}

The effect of the presence of other protein biomarkers and non-target compounds potentially co-existing with CD105 in human serum on the immunosensor response was evaluated. The amperometric responses provided by the developed immunosensors for 0 and $1 \mathrm{ng} \mathrm{mL} \mathrm{g}^{-1} \mathrm{CD} 105$ standards were measured both in the absence and in the presence of the following compounds: BSA, IgG, hemoglobin (HB), transforming growth factor beta-1 (TGF$\beta 1$ ), interleukin 6 (IL-6), interleukin 1 beta (IL-1 $\beta$ ), E-cadherin (E-Cad), uric acid (UA), ascorbic acid (AA), recombinant human ErbB2 protein (ERBB2), human p53 (p53), cadherin 17R (Cad17R) and fibroblast growth factor receptor 4 (FGFR4). The tested concentrations of these compounds were those reported as their normal levels in human serum. Fig. 5 shows clearly as no significant differences occurred between the currents measured in the absence or in the presence of these potential interferents, thus demonstrating the practical specificity of the immunosensor for the determination of CD105.

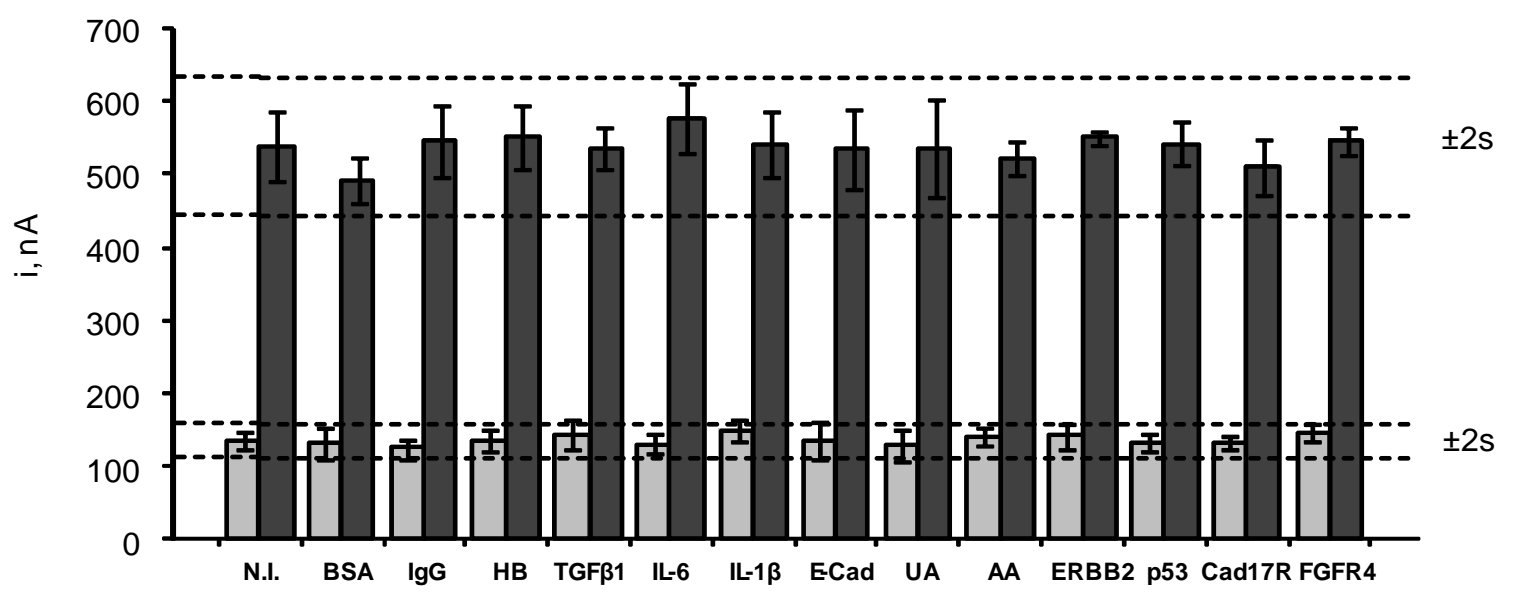

Fig. 5. Amperometric responses measured with the poly-HRP-Strept-Biotin-anti-CD105CD105-anti-CD105-pPPA/SPCEs for 0 (grey bars) and 1 (black bars) ng $\mathrm{mL}^{-1} \mathrm{CD} 105$ standards prepared in the presence of $5 \mathrm{mg} \mathrm{mL}^{-1} \mathrm{BSA}, 1 \mathrm{mg} \mathrm{mL}^{-1} \mathrm{IgG}, 5 \mathrm{mg} \mathrm{mL}^{-1} \mathrm{HB}, 50 \mathrm{ng}$ $\mathrm{mL}^{-1}$ TGF- $\beta 1,300 \mathrm{pg} \mathrm{mL}^{-1} \mathrm{IL}-6,50 \mathrm{pg} \mathrm{mL}^{-1} \mathrm{IL}-1 \beta, 55 \mathrm{ng} \mathrm{mL} \mathrm{L}^{-1}$ E-Cad, $100 \mu \mathrm{g} \mathrm{mL}^{-1} \mathrm{UA}, 7$ 


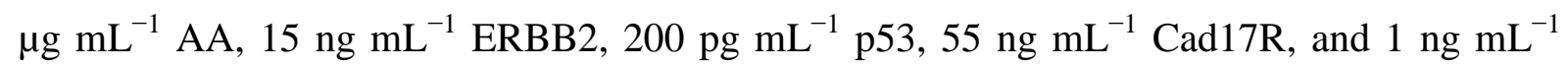
FGFR4. Error bars estimated as triple of the standard deviation $(n=3)$.

\subsection{Determination of CD105 in human serum samples}

The real usefulness of the developed immunosensors was verified by analyzing human serum samples collected from healthy individuals (3) and patients of colorectal (3), breast (3) and lung (3) cancer, as well as from treated (2) and untreated (2) epidermolysis bullosa patients. The potential existence of a matrix effect was evaluated by comparing the slope of the calibrations plot prepared with CD105 standards in buffered solutions with that constructed in serum after applying different dilution ratios $(0,1: 2,1: 3,1: 5,1: 10,1: 50$, and 1:100) with 0.01 M PBS of $\mathrm{pH}$ 7.4. The comparison of the slope of the calibrations plots obtained for CD105 standards prepared in buffered solutions $(476 \pm 12)$ and 50-times diluted healthy (475 \pm 8$)$, breast cancer $(477 \pm 7)$ and untreated epidermolysis bullosa $(470 \pm 30)$ patients, exemplified that this dilution was sufficient to avoid a significant matrix effect. Therefore, quantification of CD105 could be accomplished by interpolating the currents measured in the 1:50 diluted samples into the calibration plot prepared with CD105 standards, without any other sample treatment. The results obtained by triplicate with the immunosensor were compared with those provided by an ELISA kit that uses the same immunoreagents (Table 2). As it can be seen, no apparent differences exist between the results provided by the two methods. This was verified by means of a paired samples t-test $(\alpha=0.05$; p-value $=0.3)$. The plot of the mean concentrations obtained with the immunosensors vs. those provided by the ELISA kit resulted in a linear least-squares regression graph $(r=0.998)$ with a slope value of $(1.01 \pm 0.02)$ and an intercept of $(-0.8 \pm 1.0)$. The highly satisfactory correlation found with confidence intervals for the slope and intercept values (at a significance level of $\alpha=0.05$ ) including the unit and the zero values, respectively, indicated that the developed methodology exhibited no 
systematic errors. It is important to mention that the LOD achieved with the immunosensor is similar to the lowest concentration standard recommended by the ELISA kit involving the same immunoreagents (140 vs $125 \mathrm{pg} \mathrm{mL}^{-1}$ ), However, and importantly, the immunosensor is able to perform the determination in half time than the ELISA kit (135 vs. 280 min) once the anti-CD105-pPPA/SPCEs and anti-CD105-plate were prepared and blocked, respectively. Moreover, the amperometric determination may be easily automated and implemented with portable and cost-effective instrumentation, making the developed immunosensor an attractive and user-friendly tool to monitor routinely CD105 serum levels even for decentralized analysis and more suitable than ELISA methods for bedside and emergency medicine. Additional advantages compared with the ELISA methodologies include the easy translation of the approach to determine other specific biomarkers limiting the hospital admission and stay (drastically alleviating the hospital expenses) [27,28] and the facile integration into multiplexed (similarly to the ELISA kits, it is possible to carry out 96 determinations at the same time by using the electrochemical ELISA plates marketed in recent years) platforms.

Importantly, the plot of data summarized in Table 2 (Fig. 6) visualizes clearly the differences in the CD105 concentration in the sera of healthy individuals and for cancer patients, thus supporting the relevant role of this protein as a cancer biomarker in human serum. In addition, it was also possible to discriminate between patients suffering epidermolysis bullosa, which exhibited also elevated CD105 levels in serum. 
Table 2

Determination of CD105 (in $\mathrm{ng} \mathrm{mL}^{-1}$ ) in human serum samples.

\begin{tabular}{|c|c|c|c|c|}
\hline \multicolumn{2}{|c|}{ Patient } & No. & $\begin{array}{l}\text { nunosen } \\
(\mathrm{n}=3)^{*}\end{array}$ & ELISA, $(n=3)^{*}$ \\
\hline \multirow{5}{*}{\multicolumn{2}{|c|}{ Healthy }} & 1 & $19 \pm 2$ & $20 \pm 2$ \\
\hline & & 2 & $12 \pm 2$ & $10 \pm 2$ \\
\hline & & 3 & $20 \pm 1$ & $20 \pm 1$ \\
\hline & & 4 & $11 \pm 1$ & $10 \pm 1$ \\
\hline & & 5 & $18 \pm 2$ & $18 \pm 2$ \\
\hline \multirow{9}{*}{ Cancer } & \multirow{3}{*}{$\begin{array}{l}\text { Colorectal } \\
\text { (CRC) }\end{array}$} & 6 & $50 \pm 4$ & $50 \pm 4$ \\
\hline & & 7 & $64 \pm 3$ & $67 \pm 5$ \\
\hline & & 8 & $57 \pm 5$ & $56 \pm 5$ \\
\hline & & 9 & $81 \pm 1$ & $82 \pm 5$ \\
\hline & Breast (BC) & 10 & $86 \pm 5$ & $89 \pm 3$ \\
\hline & & 11 & $84 \pm 4$ & $79 \pm 8$ \\
\hline & & 12 & $67 \pm 5$ & $67 \pm 6$ \\
\hline & Lung (LC) & 13 & $59 \pm 4$ & $59 \pm 3$ \\
\hline & & 14 & $58 \pm 3$ & $57 \pm 5$ \\
\hline \multirow{2}{*}{\multicolumn{2}{|c|}{ Epidermolysis bullosa (EB) }} & 15 & $71 \pm 5$ & $71 \pm 6$ \\
\hline & & 16 & $75 \pm 9$ & $76 \pm 5$ \\
\hline
\end{tabular}




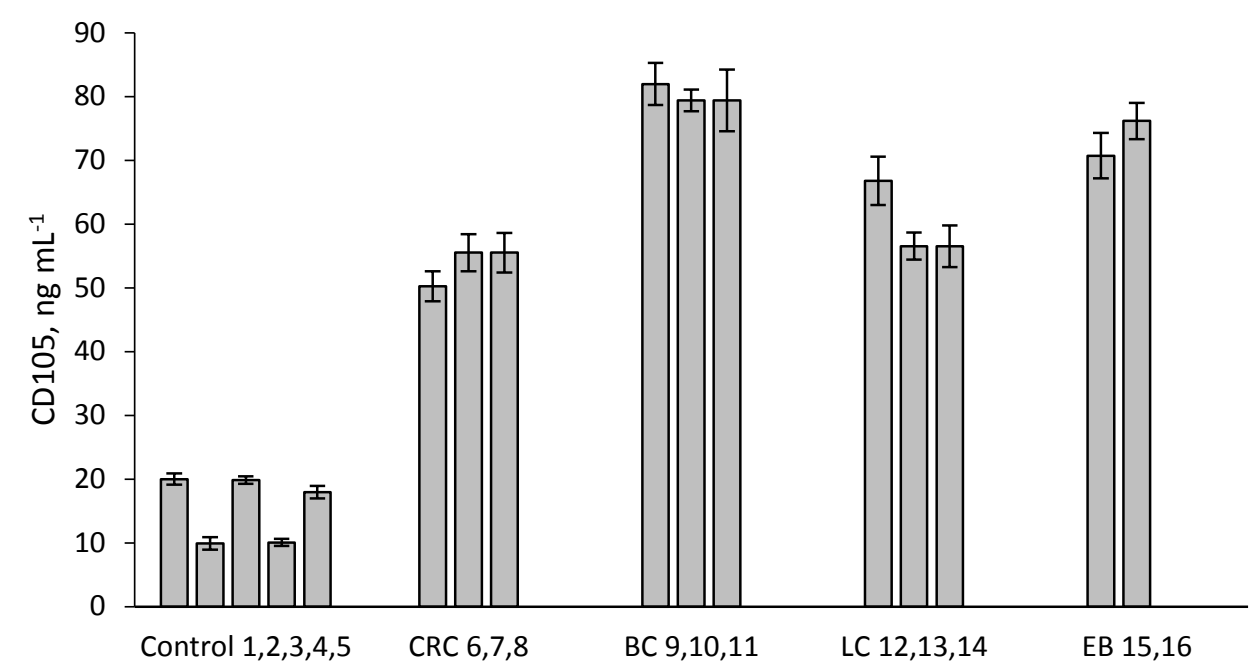

Fig. 6. CD105 concentration (in $\mathrm{ng} \mathrm{mL}^{-1}$ ) provided by the developed immunosensor in the serum samples analyzed. Error bars estimated as triple of the standard deviation $(n=3)$.

\section{Conclusions}

This work reports a novel disposable integrated inmunosensor for the determination of CD105 based on covalent immobilization of the capture antibody on pPPA-modified SPCEs using EDC/NHSS chemistry. The amperometric immunosensor exhibits a very attractive analytical performance in terms of sensitivity, selectivity and stability as well as successful applicability for the accurate determination of the endogenous content of the target protein in human serum samples from cancer and epidermolysis bullosa patients with no matrix effect upon a simple 50-times sample dilution. The simplicity, rapidity, affordability, portability, and multiplexing ability of the developed methodology to provide quantification of CD105 in small amounts of scarcely treated complex samples make it more compatible with the clinical requirements than the current available methods to bring the determination of this biomarker from the laboratory into point-of-care testing devices to perform on-site determinations even in resource-limited settings (outpatient clinics, near patients' beds or at home). Indeed, the feasibility to perform routine screening of this relevant target transmembrane glycoprotein (or other candidate 
circulating protein biomarkers) can allow continued investigation of its clinical relevance/role in physiological/pathological conditions improving patient management, therapy efficiency and high relevant diseases outcomes.

\section{Acknowledgments}

The financial support of the Spanish Ministerio de Economía y Competitividad CTQ2015-70023-R and CTQ2015-64402-C2-1-R, and SAF2014-53209-R Research Projects, the PI17CIII/00045 research project from AESI and the NANOAVANSENS Program from the Comunidad de Madrid (S2013/MT-3029). E.M-P. acknowledged Spanish Ministry (Economía, Industria y competitividad) for Juan de la Cierva-Formación fellow.

\section{References}

[1] S. E. Duff, C. Li, J.M. Garland, S. Kumar, CD105 is important for angiogenesis: evidence and potential applications, The FASEB Journal 17 (2003) 984-992.

[2] F. Nassiri, M.D. Cusimano, B.W. Scheithauer, F. Rotondo, A. Fazio, G.M. Yousef, L.V. Syro, K. Kovacs, R.V. Lloyd, Endoglin (CD105): a review of its role in angiogenesis and tumor diagnosis, progression and therapy, Anticancer Res. 31 (2011) 2283-2290.

[3] F.J. Burrows, E.J. Derbyshire, P.L. Tazzari, P. Amlot, A.F. Gazdar, S.W. King, M. Letarte, E.S. Vitetta, P.E. Thorpe, Up-regulation of endoglin on vascular endothelial cells in human solid tumors: implications for diagnosis and therapy, Clin. Cancer Res. 1 (1995) $1623-1634$.

[4] B. Bodey, S.E. Siegel, H.E. Kaiser, Overexpression of endoglin (CD105): a marker of breast carcinoma-induced neo-vascularization, Anticancer Res. 18 (1998) 3621-3628. 
[5] S. Kumar, A. Ghellal, C. Li, G. Byrne, N. Haboubi, J.M. Wang, N. Bundred, Breast carcinoma: vascular density determined using CD105 antibody correlates with tumor prognosis, Cancer Res. 59 (1999) 856-861.

[6] M. Quintanilla, J.R. Ramirez, E. Pérez-Gómez, D. Romero, B. Velasco, M. Letarte, J.M. López-Novoa, C. Bernabéu, Expression of the TGF-b coreceptor endoglin in epidermal keratinocytes and its dual role in multistage mouse skin carcinogenesis, Oncogene 22 (2003) 5976-5985.

[7] V.L. Martins, M.P. Caley, K. Moore, Z. Szentpetery, S.T. Marsh, D.F. Murrell, M.H. Kim, M. Avari, J.A. McGrath, R. Cerio, A. Kivisaari, V.M. Kähäri, K. Hodivala-Dilke, C.H. Brennan, M. Chen, J.F. Marshall, E.A. O'Toole, Suppression of TGF $\beta$ and angiogenesis by type VII collagen in cutaneous SCC, J. Natl. Cancer Inst. 108 (2015) pii: djv293. doi: 10.1093/jnci/djv293.

[8] E. Rossi, F. Sanz-Rodriguez, N. Eleno, A. Düwell, F.J. Blanco, C. Langa, L.M. Botella, C. Cabañas, J.M. López-Novoa, C. Bernaveu, Endothelial endoglin is involved in inflammation: role in leukocyte adhesion and transmigration. Blood 121 (2013) 403-415.

[9] P.K. Aggarwal, N. Chandel, V. Jain, V. Jha, The relationship between circulating endothelin-1, soluble fms-like tyrosine kinase-1 and soluble endoglin in preeclampsia, J. Hum. Hypertens. (2011) 1-6.

[10] A.E. Koch, The role of angiogenesis in rheumatoid arthritis: recent developments, Ann Rheum Dis. 59 (2000) i65-i71.

[11] C. Hümefeld, M. Mezger, J.S. Kern, A. Nysytöm, K. Brickner-Tuderman, I. Müller, R. Handgretinger, M. Röcken, One goal, different strategies - molecular and cellular approaches for the treatment of inherited skin fragility disorders, Exp. Dermat. 22 (2013) $162-167$. 
[12] I.V. Smirnov, I.V. Gryazeva, M.P. Samoylovich, L.A. Terekhina, A.A. Pinevich, O.A. Shashkova, I.Y. Krutetskaia, D.I. Sokolov, S.A. Selkov, N.N. Nikolskiy, V.B. Klimovich, Different pairs of monoclonal antibodies detect variable amounts of soluble endoglin in human blood plasma, Immunochem. Immunopathol. 2 (2016) 473-482.

[13] A.L. Gregory, G. Xu, V. Sotov, M. Letarte, Review: the enigmatic role of endoglin in the placenta, Placenta, 35 (2014) 593-599.

[14] www.thermofisher.com/order/catalog/product/EHENG

[15] www. sigmaaldrich.com /catalog/ product/ sigma/rab0171? Lang= es \& regin=ES

[16] http://www.sinobiologicalcdn.com/reagent/KIT10149.pdf

[17] S. Zeng, S. Wang, L. Wang, L. Yang, Z. Chen, Z. Liang, A novel CD105 determination system based on an ultrasensitive bioelectrochemical strategy with Pt nanoparticles, Sensors 12 (2012) 13471-13479.

[18] R.M. Torrente-Rodríguez, S. Campuzano, V. Ruiz-Valdepeñas-Montiel, M. Pedrero, M.J. Fernández-Aceñero, R. Barderas, J.M. Pingarrón, Rapid endoglin determination in serum samples using an amperometric magneto-actuated disposable immunosensing platform, J. Pharm. Biomed. Anal. 129 (2016) 288-293.

[19] N. Takahashi, R. Kawanishi-Tabata, A. Haba, M. Tabata, Y. Haruta, H. Tsai, B. K. Seon, Association of serum endoglin with metastasis in patients with colorectal, breast, and other solid tumors, and suppressive effect of chemotherapy on the serum endoglin, Clin. Cancer Res. 7 (2001) 524-532.

[20] H. Dong, X. Cao, C.M. Li, W. Hu, An in situ electrochemical surface plasmon resonance immunosensor with polypyrrole propylic acid film: Comparison between SPR and electrochemical responses from polymer formation to protein immunosensing, Biosens. Bioelectron. 23 (2008) 1055-1062. 
[21] H. Dong, C. M. Li, W. Chen, Q. Zhou, Z-X. Zeng, J.H.T. Luong, Sensitive amperometric immunosensing using polypyrrolepropylic acid films for biomolecule immobilization, Anal. Chem. 78 (2006) 7424-7431.

[22] Y. Hu, Z. Zhao, Q. Wan, Facile preparation of carbon nanotube conducting polymer network for sensitive electrochemical immunoassay of Hepatitis B surface antigen in serum, Bioelectrochem. 81 (2011) 59-64.

[23] V. Serafín, L. Agüí, P. Yáñez-Sedeño, J.M. Pingarrón, Electrochemical immunosensor for the determination of insulin-like growth factor-1 using electrodes modified with carbon nanotubes-poly(pyrrole propionic acid) hybrids, Biosens. Bioelectron. 52 (2014) 98-104.

[24] V. Serafín, L. Agüí, P. Yáñez-Sedeño, J.M. Pingarrón, Determination of prolactin hormone in serum and urine using an electrochemical immunosensor based on poly(pyrrolepropionic acid)/carbon nanotubes hybrid modified electrodes, Sens. Actuator B-Chem. 195 (2014) 494-499.

[25] V. Serafín, R.M. Torrente-Rodríguez, M. Batlle, P. García de Frutos, S. Campuzano, P. Yáñez-Sedeño, J.M. Pingarrón, Electrochemical immunosensor for receptor tyrosine kinase AXL using poly(pyrrolepropionic acid)-modified disposable electrodes, Sens. Actuator B-Chem. 240 (2017) 1251-1256.

[26] M. Eguílaz, M. Moreno-Guzmán, S. Campuzano, A. González-Cortés, P. Yáñez-Sedeño, J. M. Pingarrón, An electrochemical immunosensor for testosterone using functionalized magnetic beads and screen-printed carbon electrodes, Biosens. Bioelectron. 26 (2010) $517-522$.

[27] M. Mascini, S. Tombelli, Biosensors for biomarkers in medical diagnostics, Biomarkers 13 (2008) 637-657.

[28] F. Ricci, G. Adornetto, G. Palleschi, A review of experimental aspects of electrochemical immunosensors, Electrochim. Acta 84 (2012) 74-83. 


\section{Graphical Abstract}

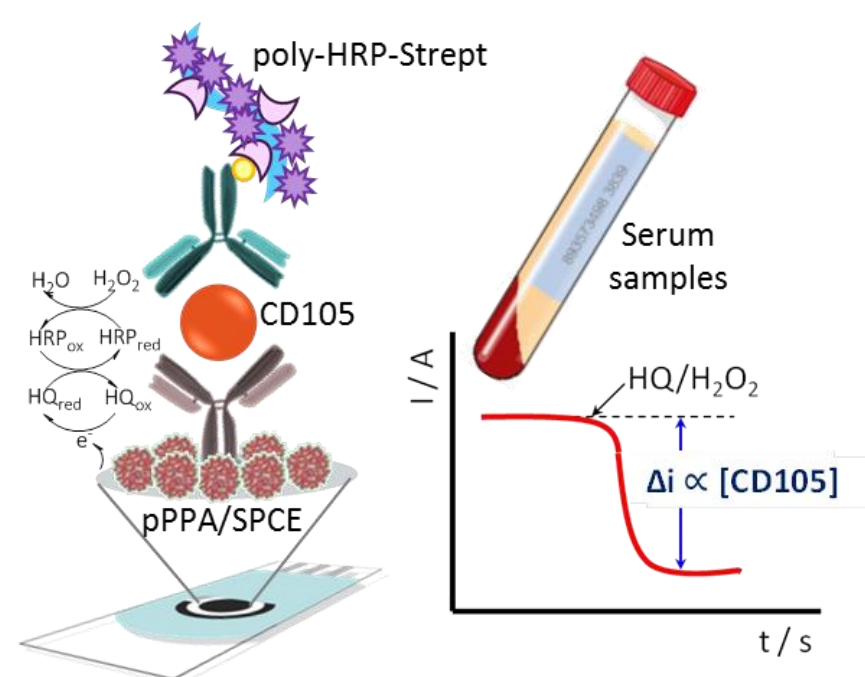

Amperometric immunosensing platform, implemented onto a disposable electrode modified with pPPA and involving a sandwich configuration for sensitive and accurate determination of CD105 in serum samples from cancer or epidermolysis bullosa patients with minimal sample treatment. 